\title{
Teleradiologie in der Schweiz?
}

\author{
Interview mit PD Dr. C. W. A. Pfirrmann, dem Präsidenten der Arbeitsgemeinschaft Teleradiologie der Schweizerischen \\ Gesellschaft für Radiologie SGR-SSR*, anlässlich des Teleradiologiekongresses vom 25. November 2004 in Zürich.
}

\begin{abstract}
SÄZ: Der Begriff Telemedizin wird in den Medien immer häufiger aufgegriffen. Im praktischen Alltag scheint die Telemedizin aber noch nicht zur Routine geworden zu sein. Trifft dies auch für die Teleradiologie zu?

PD Dr. C. W. A. Pfirrmann: Die Radiologen gehören zu den aktivsten Telemedizinanwendern in der Schweiz. Eine umfassende Erhebung bei den Schweizer Radiologen hat gezeigt, dass die Teleradiologie in den verschiedensten Einsatzbereichen routinemässig zum Einsatz kommt. Dazu gehören neben dem einfachen Bildversand an Zuweiser die Notfallversorgung von Patienten, das interaktive Besprechen von Fällen, das Einholen von Zweitmeinungen oder Expertenbeurteilungen, aber auch die Ausbildung und der Studentenunterricht. $77 \%$ der Antwortenden verwenden Teleradiologie in ihrem Alltag oder werden dies in naher Zukunft tun.
\end{abstract}

Die Schweiz hat auch in ihren Randregionen eine bestens ausgebaute medizinische Infrastruktur. Die Distanzen sind klein. Hat unter diesen Voraussetzungen die Teleradiologie in der Schweiz überhaupt einen Stellenwert?

Gerade mit der immer besser werdenden medizinischen Infrastruktur auch in Randgebieten der Schweiz nimmt die Bedeutung der Teleradiologie zu. Viele kleinere Spitäler in ländlichen Regionen werden mit Computertomographen ausgerüstet. Oft werden diese Spitäler nicht rund um die Uhr von einem Radiologen betreut. Zur Gewährleistung einer kompetenten Versorgung des Patienten werden die Bilder teleradiologisch in ein Zentrumsspital geschickt und dort vom diensthabenden Notfallradiologen beurteilt.

PD Dr. C. W. A. Pfirrmann ist Leitender Arzt Radiologie an der Uniklinik Balgrist und präsidiert seit Sommer 2003 die Arbeitsgemeinschaft Teleradiologie der SGR-SSR. Die Arbeitsgruppe hat unter anderem im Rahmen einer Dissertation die Teleradiologiekontakte der Schweizer Radiologen erforscht und führt im Auftrag der Schweizerischen Gesellschaft für Telemedizin am 25. November 2004 an der Uniklinik Balgrist eine Teleradiologietagung durch (www.balgrist.ch).
Wird die Teleradiologie auch in der interdisziplinären Zusammenarbeit bei der Notfallversorgung des Patienten eingesetzt?

Die Untersuchungen können teleradiologisch sehr schnell zur konsiliarischen Beurteilung in Spezialkliniken übermittelt werden. So kann zum Beispiel eine Computertomographie des Schädels bei einem Patienten mit Schädel-HirnTrauma zur konsiliarischen Beurteilung in ein Zentrum mit einer Neurotraumatologie übermittelt werden.
Was sind die Hardware- und Softwarevoraussetzungen für einen Teleradiologiebetrieb?

Die Bilddaten müssen in digitaler Form vorliegen. Die meisten radiologischen Bilddaten in Kliniken und Radiologieinstituten werden bereits heute digital im sogenannten DICOM-Format akquiriert. Die Bilddaten werden in einem Spital, Spitalverbund oder Zuweisernetzwerk in einem PACS (Picture Archiving and Communication System) verfügbar gemacht und mit einem RIS (Radiology Information System) verwaltet. Aus einem PACS-System können die Bilddaten in der Regel sehr einfach in ein anderes PACS-System verschickt werden. Die Daten können aber auch auf einem Server zur Verfügung gestellt werden. Von dort können Sie über ein Webinterface durch eine gesicherte Internetverbindung vom Berechtigten abgerufen werden.

Was bedeutet eigentlich DICOM, und was ist die Rolle von DICOM in der Teleradiologie? Die Abkürzung DICOM steht für «Digital Imaging and Communication in Medicine». DICOM ist ein internationales Format für radiologische Bilddaten. DICOM wurde in Zusammenarbeit zwischen dem American College of Radiology (ACR) und der National Electronics Manufactures Association (NEMA) entwickelt. Nahezu alle Hersteller von Geräten, die digitale radiologische Bilddaten produzieren (Phosphorplattenund Flatpanelsysteme für die konventionelle Röntgendiagnostik, CT, MRT, US, Szintigraphie, SPECT, PET, Scanner für das Auslesen von analogen, klassischen Filmen), unterstützen diesen Standard. Der DICOM-Standard definiert nicht nur, wie dieses Bildformat aufgebaut ist, sondern auch, wie die Geräte untereinander kommunizieren (Datenversand und -empfang). Interessierte können zusätzlich Information über http://medical.nema.org bekommen.

Was sind die personellen Voraussetzungen für einen Teleradiologiebetrieb?

In der Regel sind die Installationen ohne grosse Spezialkenntnisse ähnlich wie ein E-Mail-System $\mathrm{zu}$ bedienen. Statt Couverts zu verpacken und zur Post zu bringen, verschickt das Personal die Aufnahmen per Knopfdruck. 
Kann man sich Teleradiologie in der Arztpraxis vorstellen?

Viele Radiologieinstitute und Kliniken stellen dem Zuweiser und dem Patienten die Untersuchungen schon heute in digitaler Form auf einer CD-ROM zur Verfügung. Auf der CD-ROM sind die Bilddaten im original DICOM-Format vorhanden. Auf der CD-ROM befindet sich auch ein integrierter DICOM-Viewer zur Betrachtung der Bilder an einem normalen PC, so dass keine Software installiert werden muss. Mit der fortschreitenden Digitalisierung und Vernetzung wird die Direktübermittlung dieser Daten in die Praxis mehr und mehr Verbreitung finden. Institute in verschiedenen Regionen der Schweiz bieten bereits an, dass Untersuchungen aus der Arztpraxis von einem Webserver abgerufen werden können.

Wie gross ist eigentlich eine DICOM-Datei einer Thoraxaufnahme?

Ein konventioneller $35 \times 43$-cm-Film einer Thoraxaufnahme hat im DICOM-Format eine Grösse von etwa $8 \mathrm{MB}$. Eine typische MR-Untersuchung hat eine Grösse von 30 bis $50 \mathrm{MB}$. Eine moderne Multislice-CT-Untersuchung kann sogar eine Grösse vom mehreren Hundert MB erreichen. Die verlustfreie Kompression resultiert in einer Verkleinerung des Datenvolumens auf 50 bis $33 \%$. Nimmt man eine verlustbehaftete Kompression in Kauf, ist eine Reduktion der Datenmenge auf 1:20 oder mehr möglich. Die Grösse der Bilddaten bedingt, dass für eine vernünftige Übertragungszeit ein Breitbandanschluss vorhanden sein sollte.

Die Teleradiologie wird grosse Kosten verursachen. Gibt es wirtschaftliche Gesichtspunkte, die für den Einsatz der Teleradiologie sprechen? Eine typische MR- oder CT-Untersuchung wird auf mehreren Filmen dokumentiert. Die Filmkosten belaufen sich je nach Umfang der Untersuchung auf Fr. 20.- bis 50.-. Zusätzlich fallen Versandkosten von Fr. 6.- bis 13.- an. Diese Kosten belaufen sich also leicht auf bis zu 20\% des heutigen TARMED-Tarifes für die jeweilige Untersuchung. Diese Kosten würden beim teleradiologischen Versand weitgehend entfallen. Neben diesen konkreten Zahlen sind vielfältige, schwierig in Zahlen auszudrückende Vorteile zu erwähnen. Die elektronische Speicherung der Untersuchungen erspart die Kosten für ein physisches Archiv. Sie verhindert Filmverlust und damit viele Wiederholuntersuchungen und das äusserst nerven- und zeitraubende Bildersuchen.

Gibt es Risiken bei der Teleradiologie?

Einer der wichtigsten Aspekte ist der Datenschutz. Die radiologischen Daten müssen vor dem Zugriff durch Unberechtigte geschützt werden. Die Verbindungen zwischen Sender und Empfänger müssen abgesichert sein. So darf eine radiologische Untersuchung nicht einfach als Attachment eines E-Mails verschickt werden. Die Daten müssen zuerst verschlüsselt und anonymisiert werden, oder sie müssen über einen geeigneten Dienst (z.B. das HIN) versandt werden, der eine sichere Verschlüsselung der Daten gewährleistet.

Am 25. November 2004 findet an der Uniklinik Balgrist Zürich die Veranstaltung «Teleradiologie in der Schweiz» statt: Welche Informationen kann man von dieser Veranstaltung erwarten? Bei dieser Veranstaltung werden die aktuelle Situation der Teleradiologie in der Schweiz und verschiedene praktische Anwendungen der Teleradiologie beleuchtet und diskutiert werden. Neben der Anwendung in der medizinischen Dienstleistung werden auch Lehre und Forschung mit Beispielen vertreten sein. Die aktuelle rechtliche Situation wird ebenfalls auf den Tisch gelegt.

\section{Für welche Teilnehmer ist diese Veranstaltung von Interesse?}

Die Veranstaltung richtet sich an alle telemedizinisch interessierten Ärztinnen und Ärzte, an medizinisch-technische Röntgenassistenten/ -innen, an Informatiker/innen, an die medizintechnische Industrie, an Versicherungen und an Gesundheitsbehörden.

Wo erhält man zusätzliche Informationen, und wo kann man sich anmelden?

Weitere Informationen sind über die Website der Uniklinik Balgrist (www.balgrist.ch), Link «Kurse und Kongresse», abrufbar. Dort kann man sich auch anmelden. 